\title{
Palma de aceite Y Seguridad ALIMENTARIA EN EL CARIBE COLOMBIANO: el Caso del municipio de María La BaJA, Bolívar ${ }^{1}$
}

\author{
PALM OF OIL AND FOOD SECURITY IN THE COLOMBIAN \\ CARIBBEAN: THE CASE OF THE MUNICIPALITY OF MARÍA \\ LA BAJA, BOLÍVAR
}

*Doctor en Ciencias Sociales y Jurídicas y Magíster en Economía y Desarrollo Territorial de la Universidad de Cádiz, España. Especialista en Gestión Gerencial y Administrador Industrial de la Universidad de Cartagena, Colombia. Docente de Tiempo Completo de la Universidad de Cartagena, adscrito al programa de Administración Industrial y al Instituto Internacional de Estudios del Caribe. Director del grupo de investigación en Estudios para el Desarrollo Regional -GIDER-. E-mail: fmazaa@unicartagena.edu.co.

**Magíster en Desarrollo y Ambiente de la Universidad Tecnológica de Bolívar, Colombia. Administrador Industrial de la Universidad de Cartagena, Colombia. Coordinador De Investigación Formativa de la Universidad Tecnológica de Bolívar. Integrante de grupo de investigación en Estudios para el Desarrollo Regional -GIDERE-mail: gherreras@unitecnologica.edu.co

***Doctora (c) en Economía y Magíster en Desarrollo Económico y Políticas Públicas de la Universidad Autónoma de Madrid, España. Economista de la Universidad de Cartagena, Colombia. Directora del programa de Economía y de la Maestría en Desarrollo y Ambiente de la Universidad Tecnológica de Bolívar, Colombia. Email: tjimenez@unitecnologica.edu.co.

\author{
Por: Francisco Javier Maza Ávila* \\ Gustavo Adolfo Herrera Sebá** \\ Tania Isabel Jiménez Castilla***
}

Recibido: 13 de marzo de 2017 - Aprobado: 11 de junio de 2017

\begin{abstract}
RESUMEN
Este artículo aborda las implicaciones del cultivo de palma de aceite en la estructura productiva agrícola y la seguridad alimentaria de María la Baja, Colombia, uno de los municipios con mayor representatividad en la producción agrícola de la región Caribe y, en especial, de la palma de aceite. Para ello, se analizan las evaluaciones agropecuarias del periodo 2001-2014, así como los resultados tanto de una encuesta aplicada a productores de palma de aceite, como de una entrevista realizada a vendedores de productos agroalimentarios de la plaza de mercado municipal. Los resultados anteriores se contrastan con el análisis de la vulnerabilidad de la seguridad alimentaria desarrollado por el Departamento de Prosperidad Social -DPS- y el Programa Mundial de Alimentos -WFP-. Los resultados muestran que el crecimiento de la palma de aceite ha traído consigo una disminución importante de los cultivos de alimentos; también han aumentado la vulnerabilidad en materia de seguridad alimentaria.
\end{abstract}

Palabras clave: biocombustibles, seguridad alimentaria, vulnerabilidad, palma de aceite.

\section{ABSTRACT}

This article is about the implications of oil palm cultivation in the agricultural production structure and food security of María la Baja, Colombia, one of the municipalities with the highest representation in agricultural production in the Caribbean region, and especially palm of oil. For this, the agricultural evaluations of the period 2001-2014 are analyzed, as well as the results of both a survey applied to oil palm producers and an interview with sellers of agri-food products in the market square. The above results are contrasted with the analysis of the vulnerability of food security developed by the Department of Social Prosperity (DPS) and the World Food Program (WFP). The results show that the growth of oil palm has led to a significant decline in food crops; food security vulnerability has also increased.

Keywords: biofuels, food security, vulnerability, palm oil. 


\section{Introducción}

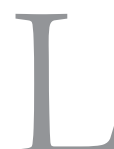

a palma de aceite -también llamada palma africana- es un cultivo de tardío rendimiento utilizado como materia prima para diversos productos de la industria alimenticia y cosmética. Se cultiva principalmente en países tropicales y, según la Food and Agriculture Organization of the United Nations -FAO- tiene como principales productores en el mundo a Indonesia y Malasia -quienes al 2014 aportaban el 85\% de la producción mundial-, constituyendo una industria relevante en su estructura económica. Colombia, por su parte, se consolida al 2014 como el cuarto mayor productor en el mundo. La palma de aceite, al igual que otros cultivos como la soya, la caña de azúcar, el maíz, entre otros, son también utilizados para la producción de biocombustibles, por ello también se les conoce como cultivos energéticos. Los biocombustibles derivados de estos cultivos agrícolas son denominados de primera generación, ya que su principal input de producción es la tierra $\mathrm{y}$, por tanto, poseen dinámicas productivas diferentes a los combustibles de fuentes fósiles (Doruk Özdemir, Härdtlein \& Eltrop, 2009).

El incremento en la demanda de biocombustibles ha respondido a una política energética mundial en pleno proceso de transformación y evolución, resultado del aumento en los precios internacionales del crudo de petróleo en los últimos años -precios en descenso durante la redacción de este artículo-, y la necesidad de disminuir las emisiones de gases de efecto invernadero -GEI- para mitigar el cambio climático. Estados Unidos, Francia, Alemania y Brasil se encuentran entre los países que han modificado sus matrices energéticas y han incluido a los biocombustibles como productos estratégicos, lo que ha derivado su creciente demanda (Rathmann, Szklo \& Schaeffer, 2010; Koh \& Ghazoul, 2008). Dicha demanda de biocombustibles ha conllevado a la incorporación de nuevas extensiones de tierras para su producción, tendencia presentada en diferentes regiones y países. Lo anterior ha sido un fenómeno de gran relevancia en el panorama político y ambiental en el mundo, debido a los impactos reales y potenciales derivados de la producción de biocombustibles a partir de cultivos agrícolas como la palma de aceite. Rathman, et. al. (2010) considera que el incremento de las tasas de producción de cultivos energéticos representa un cambio en el uso de suelo que afecta la producción de alimentos $y$, en consecuencia, genera un debate profundo sobre la necesidad de proveer de comida a la humanidad, frente los beneficios por rendimientos económicos que los agricultores reciben al incorporar sus tierras a la producción de agro combustibles.
1 Este artículo se deriva del proyecto de maestría titulado "Implicaciones de la palma de aceite en la estructura productiva agrícola y la seguridad alimentaria del municipio de María la Baja - Bolívar. Una versión preliminar fue presentada, como ponencia, en el XII Seminario Internacional de Estudios del Caribe, realizado en el año 2015. 
Para el caso de Colombia, la competencia por el uso del suelo para cultivos energéticos frente a cultivos alimentarios es una realidad, debido al incremento de la producción de palma de aceite en diferentes regiones el país y al rol protagónico que se le ha adjudicado como medio para superar la pobreza rural. Según el Documento Conpes N 3477 de 2007 (DNP, 2007), el cultivo de palma de aceite ha sido el que mayor crecimiento en hectáreas ha registrado en los últimos años en Colombia, y es uno de los productos que mayor apoyo recibe por parte del gobierno nacional. Por solo mencionar un ejemplo, para el año 2010 el Gobierno Nacional, a través de dicho Conpes, fijó la meta de incrementar el área sembrada de palma en el país a mínimo 422.000 hectáreas (WWF, 2009). No obstante, las cifras de Federación Nacional de Cultivadores de Palma de Aceite -Fedepalma-, para el año 2011 ya se registraban 427.367 hectáreas sembradas en todo el territorio nacional, sobrepasando rápidamente la meta planteada (Fedepalma, 2011). Para el año 2014 -último año con información disponible-, en Colombia se cosechan 1.278.125,53 hectáreas de palma de aceite, representando un crecimiento del 171,4\% con respecto al año 2000, mientras que el crecimiento de la producción, situada 400.193,59 toneladas, ha sido del 167,42\% en ese mismo periodo.

Concretamente, la producción de palma de aceite en Colombia se encuentra concentrada en cuatro zonas -Oriental, Norte, Central y Sur-occidental-, destacándose por su producción los municipios del Meta, Casanare, Cesar, Santander, Magdalena y Bolívar quienes, en conjunto, representan el 89,64\% de las hectáreas cosechadas del país, con destino mayoritario a la producción de aceite para biocombustibles. Se puede observar que Meta y Casanare son los departamentos con la mayor cantidad de hectáreas cosechadas de palma $-37,48 \%$ y $13,37 \%$, respectivamente-. Por su parte, los departamentos de la región Caribe aportan, en conjunto, el 28,26\% del total de áreas cosechadas y el 30,37\% de las toneladas producidas. De manera desagregada, se observa un crecimiento significativo de los municipios que registran cultivos de palma de aceite en el periodo 2001-2014. Para el primer año de la serie, se registraban apenas 66 municipios productores, mientras que, para el año 2014, ya 108 municipios tenían cultivos de palma de aceite, significando un crecimiento del 63,64\% (Tabla 1).

Ante la dinámica expansiva del cultivo de palma de aceite en Colombia, varios han sido los temores despertados. Por un lado, preocupa que el cultivo se desarrolle sin planificación en áreas no aptas o con restricciones de uso de suelo, sobrepasando las fronteras agrícolas y poniendo en riesgo ecosistemas 
Tabla 1. Cultivo de palma de aceite en Colombia por áreas cosechadas, producción y rendimiento. Año 2014

\begin{tabular}{|c|c|c|c|c|c|}
\hline Departamento & $\begin{array}{l}\text { Área Cos. } \\
\text { (Hectáreas) }\end{array}$ & $\begin{array}{c}\text { Participación } \\
\text { Área Cos. } \\
\text { Nacional (\%) }\end{array}$ & $\begin{array}{l}\text { Producción } \\
\text { (Toneladas) }\end{array}$ & $\begin{array}{c}\text { Participación } \\
\text { Producción } \\
\text { Nacional (\%) }\end{array}$ & $\begin{array}{l}\text { Rendimiento } \\
\text { (Ton/ha) }\end{array}$ \\
\hline META & $150.000,00$ & 37,48 & $456.730,18$ & 35,73 & 3,04 \\
\hline CASANARE & $53.503,94$ & 13,37 & $188.378,09$ & 14,74 & 3,52 \\
\hline CESAR & $45.022,00$ & 11,25 & $155.789,16$ & 12,19 & 3,46 \\
\hline SANTANDER & $44.237,93$ & 11,05 & $136.283,35$ & 10,66 & 3,08 \\
\hline MAGDALENA & $37.835,00$ & 9,45 & $130.264,00$ & 10,19 & 3,44 \\
\hline BOLÍVAR & $28.139,00$ & 7,03 & $95.228,90$ & 7,45 & 3,38 \\
\hline NORTE DE SANTANDER & $19.717,00$ & 4,93 & $58.376,20$ & 4,57 & 2,96 \\
\hline NARIÑO & $9.702,00$ & 2,42 & $19.404,88$ & 1,52 & 2,00 \\
\hline CUNDINAMARCA & $3.626,00$ & 0,91 & $11.276,86$ & 0,88 & 3,11 \\
\hline OTROS DEPARTAMENTOS & $1.950,00$ & 0,49 & $5.982,00$ & 0,47 & 3,07 \\
\hline VICHADA & $1.950,00$ & 0,49 & $5.982,00$ & 0,47 & 3,07 \\
\hline LA GUAJIRA & $1.154,00$ & 0,29 & $3.807,10$ & 0,30 & 3,30 \\
\hline ANTIOQUIA & 961,00 & 0,24 & $3.375,42$ & 0,26 & 3,51 \\
\hline CAUCA & 659,72 & 0,16 & $2.256,25$ & 0,18 & 3,42 \\
\hline CÓRDOBA & 410,00 & 0,10 & $1.326,30$ & 0,10 & 3,23 \\
\hline CAQUETÁ & 400,00 & 0,10 & $1.120,00$ & 0,09 & 2,80 \\
\hline CHOCÓ & 336,00 & 0,08 & 672,00 & 0,05 & 2,00 \\
\hline SUCRE & 302,00 & 0,08 & $1.032,84$ & 0,08 & 3,42 \\
\hline ATLÁNTICO & 220,00 & 0,05 & 660,00 & 0,05 & 3,00 \\
\hline TOLIMA & 48,00 & 0,01 & 120,00 & 0,01 & 2,50 \\
\hline ARAUCA & 20,00 & 0,00 & 60,00 & 0,00 & 3,00 \\
\hline TOTAL & $400.193,59$ & 100 & $1.278 .125,53$ & 100 & 3,06 \\
\hline
\end{tabular}

Fuente: Elaboración propia, a partir de Evaluaciones Agropecuarias del Ministerio de Agricultura y Desarrollo Rural

estratégicos como bosques secos y tropicales y su biodiversidad. Por otro lado, existe una preocupación especial por los procesos de concentración de la propiedad de la tierra, como también de las condiciones laborales inadecuadas de pequeños productores y asociaciones en diversas zonas del país y, en especial, en la afectación de la seguridad alimentaria (WWF, 2009). En atención a lo anterior, el presente artículo plantea el análisis de las implicaciones que tiene el cultivo de palma de aceite en la estructura productiva agrícola y la seguridad alimentaria de los municipios productores de la región Caribe colombiana, tomando como caso de estudio el municipio de María la Baja, del 
departamento de Bolívar, uno de los municipios con mayor representatividad en la producción agrícola regional (Maza, 2012; Maza \& Pérez, 2015) y, en especial, de la palma de aceite.

Para ello, se analizan las evaluaciones agropecuarias del departamento de Bolívar del periodo 2001-2014 y los resultados de una encuesta aplicada a una muestra representativa de productores de palma de aceite, que incorporó variables para cuantificar los cambios del uso del suelo agrícola a partir de la introducción y crecimiento del cultivo, con especial énfasis en los efectos generados en los cultivos básicos alimenticios tradicionalmente producidos en el municipio. Para esta investigación se ha formulado un indicador, denominado Tasa de Sustitución de Cultivos Agrícolas por Palma de Aceite-TSCP-, que permite estimar el número de hectáreas de cultivos agrícolas sustituidas con la finalidad de darle paso a la producción de palma de aceite. También se analiza la situación actual de Seguridad Alimentaria y Nutricional -SAN- del departamento de Bolívar y el municipio de María la Baja, a partir del mapeo y análisis de la vulnerabilidad de la seguridad alimentaria desarrollado por el Departamento de Prosperidad Social -DPS- y el Programa Mundial de Alimentos -WFP por sus siglas en inglés-. Por último, se contrastan los resultados anteriores con la apreciación que sobre la situación de seguridad alimentaria tienen los vendedores -ambulantes y estacionarios- de la plaza de mercado del municipio.

\section{Consideraciones teóricas sobre la palma de aceite y la seguridad alimentaria.}

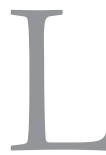

os efectos de los cultivos energéticos en la seguridad alimentaria de un territorio han sido abordados por diversos investigadores a nivel mundial. Rathman, et. al. (2010) considera que el incremento de las tasas de producción de cultivos energéticos representa un cambio en el uso de suelo que afecta la producción de alimentos y, en consecuencia, genera un debate profundo sobre la necesidad de proveer de comida a la humanidad versus los beneficios por rendimientos económicos que los agricultores reciben al incorporar sus tierras a la producción de agrocombustibles. Por su parte, Wicke, Sikkema, Dornburg \& Faaij (2010) han estudiado los efectos del cultivo de palma en los cambios de uso de suelo de diferentes países del sudeste asiático intensivos en su producción, como es el caso de Malasia e Indonesia. Estos autores manifiestan que el actual debate sobre la sostenibilidad de la producción de palma de aceite ha sido estimulado por los cambios en el uso del suelo -LUC por sus siglas en inglés: Land Use Change- que ocurren 
cuando es convertido un bosque natural, cultivos agrícolas, u otro tipo de cultivos a plantaciones de palma de aceite. Por su parte, trabajos como el de Miyake, et. al. (2012) documentan también la existencia de conversión de cultivos agrícolas existentes a cultivos para producción de biocombustibles, principalmente en los Estados Unidos y la Unión Europea, aunque también presentes en algunos casos latinoamericanos, como Brasil. Este fenómeno ha generado, a su vez, cambios indirectos en el uso del suelo en locaciones diferentes -debido al desplazamiento de los cultivos agrícolas existentes- como también el riesgo de deforestación por pérdida de bosques para ampliar las fronteras agrícolas. Para el caso específico de Brasil, Rathmann (citado por Ajanovic, 2010; p. 2073) comenta:

"Con el inicio de la producción de etanol en Brasil, la tierra y la caña de azúcar se incorporaron como insumos en la cadena de producción de combustibles, entrando en competencia con la producción de alimentos. En consecuencia, y atraídos por los altos rendimientos en comparación con la producción tradicional, muchos agricultores se desplazaron a la producción de cultivos para producción de combustibles, resultando en la sistemática sustitución de otros cultivos por el de caña de azúcar".

Las implicaciones económicas, ambientales y sociales de estos cambios de usos de suelo derivados de la producción de agrocombustibles son diversas y tienen incidencias particulares en cada territorio. Algunos autores con posiciones más radicales que otros (Wakker, 2005; Goldemberg, Coelho \& Guardabassi, 2008), plantean que los riesgos del desarrollo de cultivos bioenergéticos, en particular el de palma de aceite, incluyen las amenazas de destrucción de bosques tropicales, pérdida de biodiversidad, prácticas ilegales, conflictos sobre usos y derechos de la tierra, violaciones de derechos humanos, disputas laborales, tratamiento injusto a pequeños agricultores y colapso de prácticas culturales ancestrales, entre otros.

Otros investigadores se han concentrado en los efectos ambientales del cambio de uso de suelo para cultivos energéticos. Ajanovic (2010), en particular, manifiesta que "cualquier bosque o pastizal que se pierde para dar paso al cultivo de materia prima de aceite, almidón o azúcares causa una enorme liberación de dióxido de carbono y la producción continua de cultivos fertilizados artificialmente libera óxido nitroso, un GEI [Gas Efecto Invernadero] casi 300 veces más contaminante que el dióxido de carbono" (p. 2073). No obstante, uno de los impactos más tangibles se relaciona con el aumento del valor de 
2 El término Commodities que se hace referencia en el trabajo corresponde a los productos o bienes genéricos que no poseen alguna diferenciación entre sí y que son, generalmente, materia prima para productos con mayor valor, como lo son el trigo, la soya, el maíz y petróleo, entre otros. la tierra en los países que han insertado los biocombustibles en su matriz energética, como es el caso de Alemania y Francia, así como el incremento en los precios de los commodities ${ }^{2}$ y alimentos que han sido sustituidos por cultivos energéticos -como el trigo y la soya- en países como Brasil y Estados Unidos (Rathmann., et al, 2010). Según investigaciones del International Food Policy Research Institute -IFPRI- el aumento del 30\% de los precios de los granos a nivel mundial entre los años 2000 y 2007 se le atribuyen a la producción de biocombustibles (IFPRI, 2009; Ravindranath, Lakshmi , Manuvie \& Manuvie, 2011). En los países en desarrollo, los incrementos en los precios de alimentos asociados a los cultivos energéticos pueden tener mayor impacto en la población con altos índices de pobreza, debido a que estos gastan gran parte de sus ingresos en alimentos básicos para su sostenimiento (Janssen \& Rutz, 2011). Lo anterior tiene serias implicaciones en el acceso a bienes agrícolas básicos, en la seguridad alimentaria -tanto en poblaciones rurales como urbanas- y en el desarrollo sostenible.

\section{Metodología}

l presente artículo, derivado de una investigación eminentemente práctica, se enfoca en el análisis de las implicaciones del cultivo de palma de aceite en la estructura productiva agrícola y la seguridad alimentaria de la región Caribe colombiana, tomando como caso de estudio el municipio de María la Baja, departamento de Bolívar, caracterizado por un incremento sustancial de sus hectáreas sembradas de palma. Para ello, primero se caracteriza la estructura productiva agrícola del municipio, en términos de áreas sembradas y cosechadas, producción y rendimiento, con especial atención a la representatividad del cultivo de palma de aceite y su dinámica productiva. Para lo anterior, se toman como referencia las estadísticas productivas de las Evaluaciones Agropecuarias, disponibles tanto en el Ministerio de Agricultura y Desarrollo Rural como en la Secretaría de Agricultura y Desarrollo Rural de Bolívar, para el periodo 2001-2013, así como la información disponible en la Federación Nacional de Cultivadores de Palma de Aceite -Fedepalma- y la Asociación de Palmicultores de María la Baja -Asopalma-.

El siguiente paso consiste en determinar la incidencia del cultivo de palma de aceite en la transformación de la estructura productiva agrícola del Municipio de María La Baja. Para ello se analizan los resultados de un cuestionario aplicado en el año 2014 -mediante la técnica de encuesta- a una muestra representativa de 228 cultivadores de palma de aceite del municipio (nivel de 
confianza $=95 \%$; Error $=5,2 \%$, con el fin de identificar variables claves que permitan explicar la existencia de transformaciones en la estructura agrícola a partir de la dinámica productiva de la palma de aceite en el municipio. Este análisis se complementa con el indicador creado para esta investigación, denominado Tasa de Sustitución de Cultivos Agrícolas por Palma de Aceite-TSCP-, el cual permite estimar el número de hectáreas de cultivos agrícolas que se han sustituido para darle paso a la producción palmera.

El último paso ha consistido en identificar estados actuales o riesgos potenciales sobre la seguridad alimentaria del municipio de María la Baja, a partir de las incidencias del cultivo de palma de aceite en su estructura productiva agrícola. Para ello se examina el estudio sobre la situación actual de Seguridad Alimentaria y Nutricional -SAN- del departamento de Bolívar y el municipio de María la Baja, a partir del mapeo y análisis de la vulnerabilidad de la seguridad alimentaria desarrollado por el Departamento de Prosperidad Social -DPS- y el Programa Mundial de Alimentos -WFP por sus siglas en inglés-. Los resultados se contrastan con la apreciación que sobre riesgos actuales y potenciales a la seguridad alimentaria tienen los vendedores -ambulantes y estacionarios- de la plaza de mercado del municipio, información obtenida a partir de la aplicación de una encuesta semi-estructurada.

\section{RESULTADOS}

\section{La palma de aceite en la estructura productiva agrícola de María la Baja}

1 municipio de María la Baja tiene una extensión territorial de 517 Km²

y se encuentra ubicado en la parte norte del departamento de Bolívar, en la Zona de Desarrollo Económico y Social-Zodes- Montes de María ${ }^{3}$. Limita con los municipios de San Juan de Nepomuceno y Mahates al este, con el municipio de San Onofre -departamento de Sucre- al oeste, al norte con el municipio de Arjona y con los municipios de El Carmen de Bolívar y San Jacinto por el sur. Tradicionalmente, el municipio se ha destacado por su vocación agrícola, las capacidades agrológicas de su suelo y su riqueza hídrica, lo que ha llevado a ser reconocido como una de las zonas con mayor potencial agrícola del Caribe colombiano (Maza, 2016) ${ }^{4}$. De hecho, son las dinámicas de producción agrícola las que constituyen la principal base económica de sus habitantes. Según los datos del Sistema de Información Territorial de los Montes de María- SITMMA- para el año 2011, la actividad agrícola concentró, aproximadamente, el 48\% de las personas ocupadas en el territorio de María la Baja (SITMMA, 2011).
3 En el año 2001, la Asamblea del departamento de Bolívar organizó el territorio departamental en seis (6) Zonas de Desarrollo Económico y Social o ZODES, siendo estas Dique, Montes de María, Mojana, Depresión Momposina, Loba y Magdalena Medio.

4 Es común referenciar a María la Baja y el resto de municipios de la Zodes Montes de María como las Despensas agrícolas del departamento de Bolívar y de la región Caribe, por cuanto abastecen a la región de productos agrícolas que se encuentran en su cesta alimentaria. 
5 Una alianza productiva consiste en "una serie de relaciones y arreglos formales entre productores agropecuarios, comercializadores y agroindustriales, organizaciones de apoyo cuyo propósito es expandir empresarialmente las áreas de cultivos de rendimiento tardío y actualizar en materia tecnológica las unidades productivas de los pequeños productores asociados" (Indepaz, 2013, p, 16)

6 El grupo Oleoflores integra un conjunto de empresas organizadas alrededor de la producción y transformación de la palma de aceite mediante procesos industriales, con presencia en gran parte de las zonas productivas de palma registrada en Colombia, mediante plantaciones y plantas de transformación.

7 Si bien este dato corresponde a las cifras oficiales publicadas por la Secretaría de Agricultura y Desarrollo Rural de Bolívar, a través de las evaluaciones agropecuarias, se estima por información no oficial que las hectáreas sembradas de palma de aceite en María la Baja son mayores a las publicadas en dichas evaluaciones. De hecho, en noticia publicada por el periódico El Universal (Cartagena), se afirma que el área sembrada de Palma de aceite en este municipio ascienden a más de 22.000 hectáreas para 2013, ostensiblemente mayor los datos publicados en las evaluaciones agropecuarias para ese año (Figueroa, 2014).
Los primeros referentes de producción de palma de aceite en María la Baja se remontan a finales de los años noventa, luego de la crisis en la producción de arroz de riego, uno de los productos de mayor relevancia en este municipio, conllevando al abandono o subutilización de las tierras destinadas a este cultivo y, por consiguiente, al deterioro de su sistema de riego. A partir de este evento se estableció una alianza productiva ${ }^{5}$ que buscaba recuperar la dinámica agroindustrial en la zona y reactivar la infraestructura productiva disponible. Es así como se establece un modelo asociativo de cultivo de palma de aceite, integrando a actores institucionales, empresariales y agricultores, apalancados por la presencia y experiencia de la promotora Hacienda Las Flores -empresa del grupo Oleoflores ${ }^{6}$-, la cual aportó las herramientas necesarias para implementar y fortalecer el cultivo en el municipio, fundamentado en transferencia tecnológica y de conocimientos, creación de viveros y asistencia técnica a los productores. La alianza productiva estuvo cimentada, desde el inicio, en la creación de la Asociación de Palmicultores del Distrito de Riego de María la Baja -Asopalma- como organismo integrador y gestor de este modelo productivo (Aguilera, 2002; Aguilera, 2014). A su vez, la presencia de la planta extractora de aceite de palma, la cual se encuentra en funcionamiento desde el año 2006, y administrada por Hacienda Las Flores S.A, garantiza la compra efectiva del fruto de palma producido y absorbe la producción de la zona.

En la actualidad, la palma de aceite es el cultivo con mayor representatividad en la estructura productiva agrícola del municipio, pues concentra el 52,4\% del área total sembrada y el 53,9\% del área cosechada, así como el 42,7\% de la producción total, en toneladas ${ }^{7}$. A su vez, este monocultivo representa el 92,6\% del total de hectáreas concernientes a los cultivos de tipo permanente (Tabla 2). Tan importante ha sido la penetración de la palma de aceite en María la Baja, que hoy día es comúnmente referenciado a lo largo y ancho del municipio la influencia que este cultivo ha tenido en los cambios sustanciales de su paisaje productivo.

Desde el 2001, el crecimiento del cultivo de palma de aceite ha sido vertiginoso, en detrimento de otros cultivos tradicionales. Entre el 2001 y 2005, las hectáreas sembradas de palma crecieron en un 496,5\%, a una tasa de crecimiento promedio anual del 66,3\%, mientras que el resto de los principales cultivos registraron decrecimientos promedio del 39,2\%, a excepción del arroz de riego que creció un 67,9\% en esos años. Entre los años 2005 y 2014, la palma creció en menor proporción que el periodo 2001-2005, aumentando sus hectáreas sembradas en $224 \%$, creciendo a una tasa promedio anual de 
Tabla 2. Estructura productiva agrícola del municipio de María la Baja. Año 2014

\begin{tabular}{|c|c|c|c|c|c|c|c|}
\hline Etiquetas de fila & $\begin{array}{c}\text { Área } \\
\text { Sembrada } \\
\text { (ha) }\end{array}$ & $\begin{array}{c}\text { \% Área } \\
\text { sembrada } \\
\text { Total } \\
\text { Agrícola }\end{array}$ & $\begin{array}{c}\text { Área } \\
\text { Cosechada } \\
\text { (ha) }\end{array}$ & $\begin{array}{c}\text { \% Área } \\
\text { Cosechada } \\
\text { Total } \\
\text { Agrícola }\end{array}$ & $\begin{array}{c}\text { Producción } \\
\text { (t) }\end{array}$ & $\begin{array}{c}\% \\
\text { Producción } \\
\text { Total } \\
\text { Agrícola }\end{array}$ & $\begin{array}{l}\text { Promedio de } \\
\text { Rendimiento }\end{array}$ \\
\hline PALMA DE ACEITE & $9.800,0$ & $52,4 \%$ & $7.350,0$ & $53,9 \%$ & $28.665,0$ & $42,7 \%$ & 3,9 \\
\hline MAIZ & $5.200,0$ & $27,8 \%$ & $3.400,0$ & $24,9 \%$ & $12.300,0$ & $18,3 \%$ & 2,7 \\
\hline ARROZ & $2.184,6$ & $11,7 \%$ & $1.745,9$ & $12,8 \%$ & $9.980,3$ & $14,9 \%$ & 5,8 \\
\hline PLATANO & 800,0 & $4,3 \%$ & 530,0 & $3,9 \%$ & $7.420,0$ & $11,0 \%$ & 14,0 \\
\hline FRIJOL & 170,0 & $0,9 \%$ & 170,0 & $1,2 \%$ & 238,2 & $0,4 \%$ & 1,1 \\
\hline ÑAME & 170,0 & $0,9 \%$ & 170,0 & $1,2 \%$ & $3.400,0$ & $5,1 \%$ & 20,0 \\
\hline YUCA & 160,0 & $0,9 \%$ & 100,0 & $0,7 \%$ & $1.400,0$ & $2,1 \%$ & 14,0 \\
\hline GUAYABA & 100,0 & $0,5 \%$ & 100,0 & $0,7 \%$ & $2.500,0$ & $3,7 \%$ & 25,0 \\
\hline CACAO & 60,0 & $0,3 \%$ & 30,0 & $0,2 \%$ & 21,0 & $0,0 \%$ & 0,7 \\
\hline PAPAYA & 30,0 & $0,2 \%$ & 25,0 & $0,2 \%$ & $1.000,0$ & $1,5 \%$ & 40,0 \\
\hline AJí & 30,0 & $0,2 \%$ & 20,0 & $0,1 \%$ & 240,0 & $0,4 \%$ & 12,0 \\
\hline Total general & $18.704,6$ & $100 \%$ & $13.640,9$ & $100 \%$ & $67.164,5$ & $100 \%$ & 9,6 \\
\hline
\end{tabular}

Fuente: Elaboración propia, a partir de Evaluaciones Agropecuarias del Ministerio de Agricultura y Desarrollo Rural

16,5\%. Si bien disminuye la velocidad de crecimiento después del 2005, de igual manera se registra un crecimiento mayor que el consolidado nacional para dicho periodo, el cual fue calculado en $76,6 \%$, con una tasa de crecimiento promedio anual del 7,7\%. Cabe resaltar que a mitad de la década anterior entró en vigencia la Ley 939 de $2004^{8}$-modificatoria de la Ley 818 de 2003que brinda beneficios de exención de la renta para los ingresos derivados del aprovechamiento de cultivos de tardío rendimiento por los 10 años siguientes, a partir del inicio de la producción. Si bien estos beneficios tributarios incluyen los cultivos de cacao, cítricos, frutales y palma de aceite, este último cultivo presenta la mayor dinámica productiva en el territorio de María la Baja, por encima inclusive del cacao, que también hace presencia dentro de la estructura productiva del municipio dentro del periodo de análisis (Figura 1).

El cultivo de palma de aceite también ha reconfigurado la dinámica de las actividades de cultivo en el municipio. Los resultados del cuestionario aplicado a productores de palma de aceite de María la Baja arrojaron que el 86,8\% de ellos son nativos del municipio y solo el 13,2\% son de otros municipios cercanos o de la región Caribe colombiana. El 94,3\% de estos cultivadores de palma de aceite poseen unidades productivas propias, mientras que el 5,7\% restante arriendan las tierras donde registran la producción. Cabe resaltar que el

8 Reglamentada por los decretos 3492 de 2007, 2629 de 2007; 1970 de 2005 


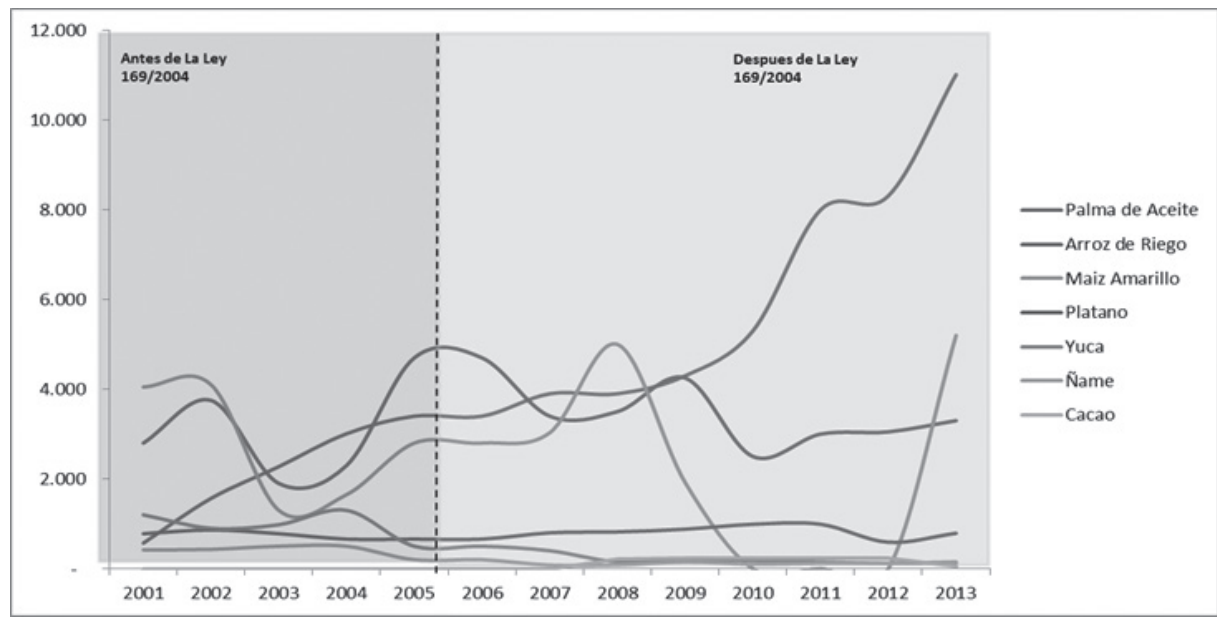

Figura 1. Comportamiento hectáreas sembrada de principales cultivos agrícolas de María la Baja. 2001-2013

Fuente: Elaboración propia, a partir de Evaluaciones Agropecuarias del Ministerio de Agricultura y Desarrollo Rural
$63,2 \%$ de estos registran entre 7 y 15 años de producción continua de palma de aceite. Si esto se traduce en términos promedios, se estima que el tiempo promedio que cada productor lleva produciendo palma de aceite es de 9 años aproximadamente. El negocio de la palma está fundamentado en la participación de minifundios y pequeñas parcelas productivas que, mediante un modelo asociativo, establecen en sus unidades el cultivo y, de esta manera, se aumenta la capacidad global de producción. En María la Baja, el 35,1\% de los predios que corresponden a unidades productivas de palma de aceite tienen una extensión menor a 5 hectáreas -minifundio- y el 53,6\% poseen predios entre 6 y 20 hectáreas de extensión -pequeño-. Las unidades de mediano y gran tamaño concentran el $5,3 \%$ y el $7 \%$ del total de predios productores, respectivamente.

De acuerdo al uso del suelo de las unidades productivas antes de iniciar la producción de palma de aceite, se puede observar que el 59,7\% de estos predios se dedicaban a la producción de cultivos agrícolas -tales como arroz, maíz, yuca, plátano, ñame, frutales, frijol, sorgo, ají, ajonjolí, tabaco y aguacate-, mientras que el 38,7\% de dichos predios eran empleados en actividades de ganadería. En particular, el 44\% de las hectáreas establecidas el año anterior al inicio del cultivo de palma correspondían al cultivo de arroz, mientras que el 21\% estaban asociadas a la producción de maíz, 15,2\% al cultivo de yuca, a la vez que el plátano y ñame concentraron el 9,6\% y 4,6\% de las hectáreas registradas, respectivamente. En una menor proporción se contabilizaron hectáreas de frutales -mango, guayaba, sapote, papaya, entre otros-, frijol, sorgo, ají, ajonjolí, tabaco y aguacate, las cuales participan, de manera conjunta, con el 5,7\% de la distribución.

Para efectos de cuantificar las implicaciones de la palma de aceite en la estructura agrícola municipal, para esta investigación se diseñó y calculó el indicador denominado Tasa de Sustitución de Cultivos Agrícolas por Palma 
de Aceite -TSCP- la cual permite estimar el número de hectáreas de cultivos agrícolas que se han sustituido para darle paso a la producción palmera durante cada año y de manera acumulada. Para ello, se asume como periodo de análisis el comprendido entre el año 2001 y el 2013, en especial, por la calidad de la información reportada. En ese sentido, se estimó en primera medida el crecimiento bruto en hectáreas de palma de aceite periodo a periodo, entendiéndose este como la diferencia entre la producción registrada en cada corte de producción, con referencia al año inmediatamente anterior (Tabla 3).

Tabla 3. Cálculo crecimiento bruto palma de aceite. Periodo 2001-2013

\begin{tabular}{|l|c|c|c|c|c|c|c|c|c|c|c|c|c|}
\hline \multicolumn{1}{|c|}{ Periodo } & $\mathbf{2 0 0 1}$ & $\mathbf{2 0 0 2}$ & $\mathbf{2 0 0 3}$ & $\mathbf{2 0 0 4}$ & $\mathbf{2 0 0 5}$ & $\mathbf{2 0 0 6}$ & $\mathbf{2 0 0 7}$ & $\mathbf{2 0 0 8}$ & $\mathbf{2 0 0 9}$ & $\mathbf{2 0 1 0}$ & $\mathbf{2 0 1 1}$ & $\mathbf{2 0 1 2}$ & $\mathbf{2 0 1 3}$ \\
\hline $\begin{array}{l}\text { TOTAL HECTÁREAS } \\
\text { REGISTRADAS POR } \\
\text { ANNO }\end{array}$ & 570 & 1.567 & 2.275 & 3.008 & 3.400 & 3.400 & 3.900 & 3.900 & 4.300 & 5.300 & 8.000 & 8.310 & 11.015 \\
\hline $\begin{array}{l}\text { CRECIMIENTO } \\
\text { BRUTO POR } \\
\text { PERIODO }\end{array}$ & 570 & 997 & 708 & 733 & 392 & 0 & 500 & 0 & 400 & 1.000 & 2.700 & 310 & 2.705 \\
\hline
\end{tabular}

Fuente: Elaboración propia, a partir de Evaluaciones Agropecuarias del Ministerio de Agricultura y Desarrollo Rural

Asimismo, se tuvo en cuenta la proporción de unidades productivas que hoy cultivan palma de aceite y que anteriormente destinaban su suelo para la producción de cultivos agrícolas -59,7\% -. En consecuencia, se tomó como referencia la composición detallada de cultivos agrícolas que fueron sustituidos por palma de aceite y se calculó su peso relativo o promedio ponderado dentro de la proporción mencionada. De este modo, al multiplicar el peso relativo de cada cultivo agrícola por el crecimiento bruto de palma de aceite por periodo, se estima el total de hectáreas sustituidas -sustitución bruta total- para cada uno de estos durante cada año y acumulado dentro del periodo de análisis.

Según este cálculo, se estima que en María la Baja se han sustituido aproximadamente 6576 hectáreas de cultivos agrícolas que fueron posteriormente destinadas a la producción de palma de aceite. De los productos que se sustituyeron se incluyen varios que componen la dieta básica alimentaria de la región, como es el caso del arroz, maíz, yuca, plátano y ñame. Para el caso del arroz, se estima una sustitución de aproximadamente 2893 hectáreas durante el periodo de análisis. Para el maíz se calcula una pérdida de 1382 hectáreas, seguido por la yuca con 998 hectáreas, el plátano con 631 hectáreas y el ñame con 300 hectáreas sustituidas. Para el agregado de frutales se estima una sustitución total de 206 hectáreas, entre los que comprenden cultivos de mango, guayaba, sapote, papaya, entre otros. De igual manera, se registran hectáreas de sorgo, tabaco, ají, frijol, ajonjolí y 
9 Cabe destacar que solo 21 hectáreas de cultivos sustituidos no correspondían a productos alimenticios, como es el caso del tabaco.

10 Según el Departamento para la Prosperidad Social, esta metodología, utilizada por las Naciones Unidades a través de su programa Mundial de Alimentos -PMA- "permite clasificar la vulnerabilidad a la inseguridad alimentaria de una población y/o territorio, e identificar quiénes son los vulnerables, cuántos son y dónde se localizan. As mismo, esta herramienta permite realizar un monitoreo y seguimiento a la situación alimentaria y nutricional, identificar tendencias y perspectivas en un determinado territorio -municipio, departamento, región o país-." (DPS, 2014, p. 9). aguacate para los cuales se estimó también la magnitud de la sustitución por el cultivo de palma de aceite (Tabla 4).

En términos relativos, la TSCP es de 0,59 hectáreas, lo que significa que por cada hectárea sembrada de palma durante el periodo de estudio se han dejado de cultivar 0,59 hectáreas de cultivos agrícolas. Estas hectáreas de cultivos potencialmente sustituidas se pueden traducir en un volumen de productos primarios, principalmente alimentos, que han dejado de ofertarse en el mercado local o regional. Se calcula que, para el periodo 2001-2013, los diferentes cultivos agrícolas que han dejado de producirse a partir de su sustitución por el cultivo de palma significaron más de 47 mil toneladas, principalmente de alimentos básicos ${ }^{9}$. Se estima que este volumen de alimentos representa en promedio un 61,8\% de la producción oficial registrada por estos cultivos alimentarios durante cada periodo.

Estos resultados se acentúan al identificar una baja tasa de mezcla de cultivos alimentarios con la palma de aceite. De hecho, los cultivos extensivos o cultivos a largo plazo dedicados a la producción de biocombustibles no acostumbran a mezclarse con otros cultivos. Los resultados del ejercicio arrojan solo una pequeña proporción de unidades productivas que si tienen establecidos cultivos agrícolas, ya sea en extensiones comunes con la palma, o bien dividieron la parcela productiva en diferentes zonas, conservando algunos de los cultivos tradicionales que acostumbran a producir.

\section{Palma de aceite y seguridad alimentaria en María la Baja}

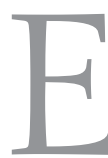
1 Departamento de Prosperidad Social -DPS- y el Programa Mundial de Alimentos -WFP, por sus siglas en inglés-, desarrollaron para el año 2014 un mapeo y Análisis de la Vulnerabilidad a la Inseguridad Alimentaria y Nutricional -VAM- ${ }^{10}$ para todos los municipios de los departamentos que componen la región Caribe colombiana (DPS \& WFP, 2014). Esta metodología se basa en dos elementos de análisis: Riesgo y Capacidad de respuesta. Este último, a su vez, se divide en tres subcomponentes, que son Disponibilidad, Acceso y Uso de los alimentos. Conceptualmente, la Vulnerabilidad a la Inseguridad Alimentaria y Nutricional está determinada por el nivel de exposición a riesgos, menos la capacidad de respuesta que puede tener una población específica para enfrentarlos -también denominado Seguridad Alimentaria Estructural-, la cual está basada en factores de disponibilidad, acceso y uso de los alimentos. El estudio determinó que, de los 46 municipios del departamento de Bolívar, el 73,3\% poseen niveles muy altos o altos de 


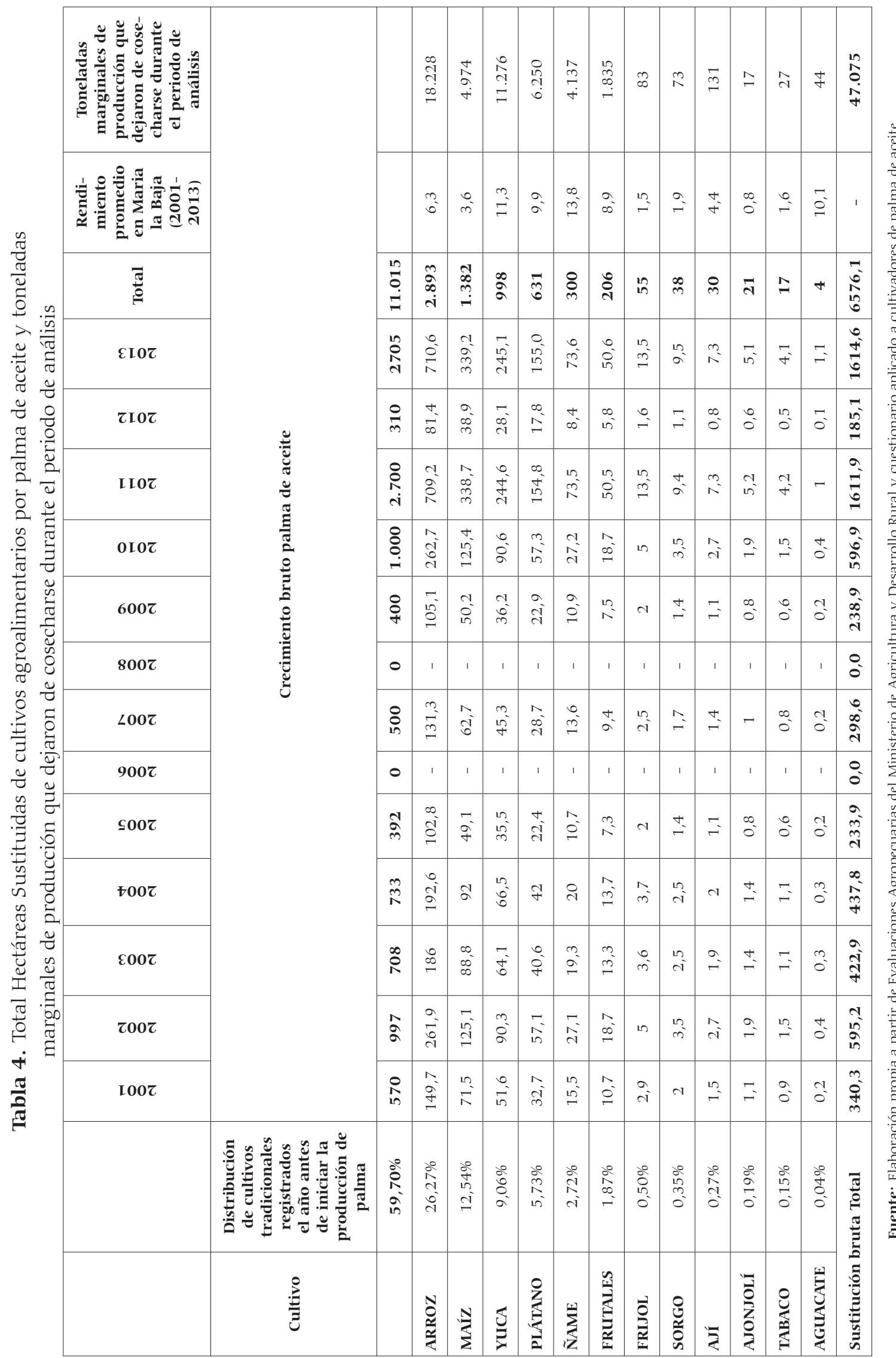


vulnerabilidad a la seguridad alimentaria y nutricional. Esta vulnerabilidad está fuertemente asociada a los altos niveles de exposiciones al riesgo, debido a la incidencia en estos territorios de factores bioclimáticos -sequías e inviernosy aspectos sociales como el nivel de ingreso, pobreza e inequidad. Según este nivel de exposición, se estimó que el 33,3\% de los municipios de Bolívar poseen niveles superiores -alto y muy alto- de riesgo para la seguridad alimentaria y nutricional.

Al analizar la distribución de municipios dentro de cada Zodes del departamento de Bolívar, se observa que la Zodes Mojana registra la mayor proporción de municipios en muy alto nivel de vulnerabilidad, seguido por Depresión Momposina, Loba y Montes de María. Esta última Zodes llama la atención por ser la única dentro de la estructura sociopolítica del departamento que posee todos sus municipios en condiciones superiores de Vulnerabilidad a la Seguridad Alimentaria. El municipio de María la Baja, en particular, es uno de los territorios con peor desempeño a nivel departamental a partir de su vulnerabilidad y nivel de riesgo alimentario, siendo uno de los tres territorios de la Zodes Montes de María bajo esta categorización. La Tabla 5 muestra los resultados cualitativos de Vulnerabilidad a la Inseguridad Alimentaria, Riesgo y Capacidad de respuesta para cada municipio montemariano.

Tabla 5. Niveles de Vulnerabilidad, Riesgo y Capacidad de respuesta por municipios de la Zodes Montes de María

\begin{tabular}{|c|c|c|c|c|c|c|c|}
\hline \multirow{2}{*}{ Municipio } & \multirow{2}{*}{$\begin{array}{l}\text { Nivel } \\
\text { VAM }\end{array}$} & \multirow{2}{*}{$\begin{array}{l}\text { Vulnerabilidad } \\
\text { a la Inseguridad } \\
\text { Alimentaria }\end{array}$} & \multirow{2}{*}{ Riesgo } & \multicolumn{4}{|c|}{ Capacidad de respuesta } \\
\hline & & & & General & Disponibilidad & Acceso & Uso \\
\hline EL GUAMO & 4 & Alta & Alto & Baja & Media & Baja & Media \\
\hline SAN JACINTO & 4 & Alta & Alto & Baja & Media & Media & $\begin{array}{l}\text { Muy } \\
\text { Baja }\end{array}$ \\
\hline $\begin{array}{l}\text { SAN JUAN } \\
\text { NEPOMUCENO }\end{array}$ & 4 & Alta & Alto & Media & Media & Media & Baja \\
\hline ZAMBRANO & 4 & Alta & Muy Alto & Media & Media & Media & Alta \\
\hline CÓRDOBA & 5 & Muy Alta & Muy Alto & Baja & Baja & Muy Baja & Baja \\
\hline $\begin{array}{l}\text { EL CARMEN DE } \\
\text { BOLÍVAR }\end{array}$ & 5 & Muy Alta & Muy Alto & Baja & Baja & Baja & Baja \\
\hline MARÍA LA BAJA & 5 & Muy Alta & Alto & Baja & Media & Baja & $\begin{array}{l}\text { Muy } \\
\text { Baja }\end{array}$ \\
\hline
\end{tabular}

Fuente: Elaboración propia a partir de DPS \& WFP (2014)

Del mismo modo, DPS y WFP determinaron la canasta usual de alimentos para la región de los Montes de María, Sierra Nevada de Santa Marta y la 
Serranía del Perijá (DPS \& WFP, 2014b). En esta se observan 16 alimentos que constituyen la dieta básica promedio para la región, principalmente con un alto índice energético. Uno de los alimentos que mayor peso relativo tiene en la canasta usual -si se tiene en cuenta los costos de compra- es el arroz. Igualmente, otros de los alimentos que hacen parte de la canasta usual de la región son la yuca y el plátano verde. A partir de la canasta usual, se recomendaron una serie de alimentos que deben ser consumidos por semana, de acuerdo con las cantidades de calorías y proteínas necesarias para alcanzar los umbrales mínimos nutricionales. Entre los alimentos que en mayor cantidad -en gramos- se exhortan a consumir por hogar en la región, con el fin de conseguir niveles óptimos de seguridad alimentaria y nutricional de sus pobladores, se destacan la leche, el arroz, el plátano, la yuca y el ñame.

Cabe resaltar, sin embargo, que gran parte de los productos alimenticios que componen esta canasta recomendada debido a su aporte energético y nutricional, son aquellos que fueron identificados como los cultivos alimentarios sustituidos, en mayor medida por la dinámica productiva de la palma de aceite. Esta situación deriva en un riesgo significativo para la seguridad alimentaria de la región y, específicamente, para el municipio de María la Baja. El arroz, que ocupa el segundo lugar de importancia en la canasta recomendada de la región según la cantidad aconsejada para su consumo, ocupa a su vez el primer lugar de los cultivos que fueron sustituidos para establecer la producción de palma de aceite. Del mismo modo, el plátano, el maíz -en forma de harina-, el ñame y la yuca, tienen un peso significativo en las cantidades nutricionales recomendadas para consumo, e igualmente fueron determinados como alimentos sustituidos de manera significativa por la palma de aceite en María la Baja (Tabla 6).

\section{Percepción de la seguridad alimentaria en María la Baja}

T a percepción de la seguridad alimentaria es entendida en este artículo como el nivel de conocimiento y sensibilización que sobre los riesgos alimentarios tiene una población específica, en un determinado territorio. Para estos efectos, se tomó en cuenta la percepción de los cultivadores de palma del municipio y de otros actores como los vendedores de alimentos básicos del mercado municipal, en referencia a los efectos que podría causar la palma de aceite en la oferta de alimentos del municipio. Es curioso observar un bajo nivel de reconocimiento de los riesgos de producir palma de aceite para la seguridad alimentaria del municipio de María la Baja. De hecho, el 75,9\% de los productores de palma afirman que no existe, en 
Tabla 6. Comparativo de los principales alimentos de la canasta recomendada para los Montes de María vs principales cultivos sustituidos por la palma de aceite en María la Baja

\begin{tabular}{|c|c|c|c|}
\hline \multicolumn{2}{|c|}{$\begin{array}{l}\text { Principales Alimentos Canasta Recomendada } \\
\text { Región Montes de María }\end{array}$} & \multicolumn{2}{|c|}{$\begin{array}{l}\text { Principales cultivos sustituidos por la } \\
\text { Palma de Aceite María La Baja }\end{array}$} \\
\hline Alimento & $\begin{array}{l}\text { Gramos por semana } \\
\text { por hogar }\end{array}$ & Cultivo & $\begin{array}{l}\text { \% de sustitución } \\
\text { ponderada }\end{array}$ \\
\hline LECHE & 7500 & ARROZ & $26.27 \%$ \\
\hline ARROZ & 2644 & MAÍZ & $12.54 \%$ \\
\hline PLÁTANO & 2500 & YUCA & $9.06 \%$ \\
\hline HARINA DE MAÍZ & 2000 & PLÁTANO & $5.73 \%$ \\
\hline ÑAME & 2000 & ÑAME & $2.72 \%$ \\
\hline PAPA & 2000 & FRUTALES & $1.87 \%$ \\
\hline YUCA & 2000 & FRIJOL & $0.50 \%$ \\
\hline POLLO & 1458 & SORGO & $0.35 \%$ \\
\hline PANELA & 1000 & AJí & $0.27 \%$ \\
\hline FRÍJOL & 1000 & AJONJOLÍ & $0.19 \%$ \\
\hline PAN & 1000 & ТАВАCO & $0.15 \%$ \\
\hline SUERO COSTEÑO & 1000 & AGUACATE & $0.04 \%$ \\
\hline
\end{tabular}

Fuente: Elaboración propia a partir de DPS (2012)

la actualidad, ninguna afectación en la disponibilidad de alimentos a causa de la producción de este cultivo en el municipio. Por su parte, aquellos que perciben un impacto negativo del cultivo consideran que es evidente la disminución de los alimentos, derivando en un aumento de los precios de los mismos. Asocian la dinámica productiva de este cultivo energético con la poca disponibilidad de tierras disponibles para la siembra de cultivos básicos, y en una menor proporción, consideran que las enfermedades que aquejan a la palma de aceite, como es el caso de la pudrición de cogollo PC-, ha afectado la producción de otros cultivos y limitado sus cosechas. Sin embargo, la proporción de cultivadores de palma que consideran una potencial afectación a futuro es mucho mayor. El 51,9\% manifiesta que, a mediano plazo, no habrá disponibilidad de alimentos en el municipio, llevando a la escasez y un consecuente aumento de precios, afectando dos dimensiones básicas de la seguridad alimentaria, como lo es la disponibilidad y el acceso de alimentos.

El aumento de los precios de los productos agrícolas es un indicador clave que permite inferir los efectos derivados por una contracción de la oferta de bienes alimentarios que, para el caso de María la Baja, está fuertemente asociado a la 
dinámica del cultivo de palma. Este efecto en el aumento de precios es percibido por los vendedores de productos agrícolas presente en el mercado municipal y otros que venden sus productos de manera ambulante ${ }^{11}$ en María la Baja, los cuales asocian directamente las causas de esta inflación con este cultivo, que ha llegado a significar un efecto inflacionario entre 200 y 300 puntos porcentuales. Asimismo, los ha llevado a tener que traer productos agrícolas directamente de la central de abastos de Cartagena de Indias:

"Un plátano vale hoy 1000, 1500 y 2000 [pesos colombianos] y antes se conseguía barato. Hoy es tan caro como en Cartagena". (Vendedor municipal 1, comunicación personal, 22 de abril de 2013).

"El plátano se vende -a día de hoy- la mano a 1.500 o 2.000 [pesos colombianos] y antes se conseguía en 500 pesos [...] ahora traemos comida de Cartagena para acá. Cuando antes la mandábamos para Cartagena". (Vendedor municipal 2, comunicación personal, 22 de abril de 2013).

En relación al efecto del cultivo de palma en la estructura productiva agrícola, se observan algunas señales que lo asocian a la sustitución de los cultivos básicos alimentarios identificados en el capítulo anterior, como es el caso de la yuca, el plátano y el frijol:

"Hay menos [alimentos] por la palma, ya que muchos han dejado la agricultura y han dejado de sembrar plátano, yuca, ñame por sembrar la palma [...] La yuca y el plátano viene poquita, el tomate y la cebolla vienen de la ciudad. Lo que ha disminuido es porque muchas tierras que cultivaban, hoy han sembrado palma". (Vendedor municipal 3, comunicación personal, 22 de abril de 2013).

"Puede ser ya que las tierras las están ocupando con un cultivo que prácticamente no es para alimentos sino para otras cosas, como el corozo [Palma]. El frijol ya no se ve como antes, como le digo, la palma ha utilizado las tierras y los cultivos han disminuido, la yuca ha disminuido bastante". (Vendedor municipal 6, comunicación personal, 22 de abril de 2013).

"Yo digo que debe ser por el corozo [Palma], ya que han usado la tierra para el cultivo del corozo y los finqueros que sembraban yuca o plátano lo han

11 Por petición propia de los vendedores, se omite el nombre de cada declaración capturada en las entrevistas. 
tumbado para sembrar corozo. (Vendedor municipal 10, comunicación personal, 22 de abril de 2013)".

Las perspectivas a futuro por parte de este grupo de vendedores redundan por las percepciones negativas a partir de la incidencia que ha tenido el monocultivo de palma de aceite en el territorio. Algunos actores manifiestan que a futuro se prevé escenarios negativos marcados por condiciones de inseguridad alimentaria y hambre en el territorio:

"Yo creo que lo que va a pasar es hambre, si se agota todo vamos a pasar es hambre [...] Nos iremos a comer los unos a los otros, nos moriremos de hambre". (Vendedor municipal 12, comunicación personal, 22 de abril de 2013)".

\section{Conclusiones}

ste artículo tuvo como objetivo analizar las implicaciones de la palma $\checkmark$ de aceite en la estructura productiva y seguridad alimentaria de la región Caribe colombiana, tomando como caso de estudio María la Baja, municipio tradicionalmente reconocido, junto con los demás que integran la región de los Montes de María, como la despensa agrícola de la región Caribe. No obstante, este municipio ha experimentado cambios profundos en su estructura productiva agrícola, asociada al crecimiento progresivo del cultivo de palma de aceite, el cual ha impactado enormemente en aquellos productos básicos alimentarios comúnmente establecidos en el territorio, tal como quedó evidenciado a partir del cálculo de la Tasa de Sustitución de Cultivos Agrícolas por Palma de Aceite-TSCP-.

El análisis de la Vulnerabilidad de la Seguridad Alimentaria, por su parte, permite afirmar que los municipios que componen el departamento de Bolívar son, en su mayoría, territorios con niveles significativos de debilidad en esta materia. Los riesgos determinantes están principalmente asociados a factores bioclimáticos como las sequías e inviernos fuertes, como también a limitaciones derivadas por el bajo nivel de ingresos y la incidencia de la pobreza en estas poblaciones. Entre las diferentes Zodes, los Montes de María la que peor situación de seguridad alimentaria presenta desde el punto de vista de su vulnerabilidad. De hecho, es la única Zodes en la que todos sus municipios son vulnerables en niveles superiores. El municipio de María la Baja es uno de los tres municipios dentro de esta Zodes que tiene una calificación de vulnerabilidad y de riesgo alto. 
Por otra parte, al analizar la canasta usual y recomendada de alimentos básicos para la región de los Montes de María -construida conjuntamente con la Sierra Nevada de Santa Marta y la Serranía del Perijá-, se observan una serie de alimentos que deben ser consumidos cada semana debido a sus características nutricionales, entre los cuales se destacan la leche, yuca, arroz, plátano y ñame. Paradójicamente, son estos alimentos los que han sido sustituidos en mayor medida por la dinámica productiva de la palma de aceite en María la Baja, por lo que se infiere que este cultivo puede incidir fuertemente en las escalas de valoración de la seguridad alimentaria a día de hoy, y posiblemente con mayor intensidad e incidencia a futuro.

Como contradicción, se observa un bajo nivel de reconocimiento, por parte de los productores de palma de aceite en el municipio de María la Baja, de los riesgos de producir este cultivo para la seguridad alimentaria. Sin embargo, una gran parte de estos consideran que la situación alimentaria va a experimentar tendencias preocupantes a futuro en el territorio. Dicha percepción es compartida por los vendedores de productos agrícolas del municipio, asociando estos escenarios con la incidencia de la palma de aceite en los cambios de la estructura productiva y alimentaria del municipio. Estos indicadores dan manifiesto de la incidencia y los riesgos actuales y potenciales de la palma de aceite en la estructura productiva agrícola de María la Baja, así como en la vulnerabilidad de sus habitantes en materia de seguridad alimentaria.

\section{REFERENCIAS BIBLIOGRÁFICAS}

Ajanovic, A. (2010). Biofuels versus food production: Does biofuels production increase food prices? Energy. Volume 36, Issue 4, April 2011, Pages 2070-2076 doi:10.1016/j.energy.2010.05.019

Aguilera, M. (2002). Palma africana en la costa Caribe: Un semillero de empresas solidarias. Documentos de Trabajo sobre Economía Regional N³0. Banco de la República.

Aguilera, M. (2014). La economía de los Montes de María. Economía \& Región, Vol. 8, No. 1, (Cartagena, junio 2014), pp. 91-141.

DNP (2007). Documento Conpes 3477: Estrategia para el desarrollo competitivo del sector palmero colombiano. Recuperado de https://www.dnp.gov.co/Portals/0/archivos/documentos/ Subdireccion/Conpes/3477.pdf

DPS \& WFP (2014). Mapeo y análisis de la vulnerabilidad a la inseguridad alimentaria y nutricional -VAM-. Convenio 035 - 2013 DPS-PMA. Colombia. Recuperado de http://www.vamcolombia. com.co/acerca-de-vam 
DPS \& WFP (2014b). Levantamiento de la información primaria que permita identificar de manera detallada a nivel local aspectos relacionados con el acceso a alimentos y uso por parte de la población de los departamentos de la región Caribe colombiana.

Doruk Özdemir, E.; Härdtlein, M. \& Eltrop, L. (2009). Land substitution effects of biofuel side products and implications on the land area requirement for EU 2020 biofuel targets. Energy Policy, (37), 2986-2996. doi:10.1016/j.enpol.2009.03.051

Fedepalma (2011). Sistema de información estadística del sector palmero. Consultado el 20 de Noviembre de 2012. Colombia.

Goldemberg, J.; Texeira Coelho, S. \& Guardabassi, P. (2008). The sustainability of ethanol production from sugarcane. Energy Policy 36, 2086- 2097. doi:10.1016/j.enpol.2008.02.028

IFPRI (2009). Respondiendo a la crisis alimentaria mundial: Tres perspectivas. Recuperado de http://www.ifpri.org/node/6523?print

Janssen, R. \& Rutz, D. (2011). Sustainability of biofuels in Latin America: Risks and opportunities. Energy Policy, 39 (2011), 5717-5725. doi:10.1016/j.enpol.2011.01.047

Koh, L. P. \& Ghazoul, J. (2008). Biofuels, biodiversity, and people: Understanding the conflicts and finding opportunities. Biological Conservation, (141), 2450-2460. doi:10.1016/j. biocon.2008.08.005

Maza Ávila, F. J. (2012). Pertinencia de la oferta educativa de la Zodes Montes de María -Colombia con sus capacidades productivas agrícolas y su potencial agroindustrial. Universidad de Cádiz.

Maza, F. \& Pérez, M. (2015). Pertinencia de la oferta educativa de la Zodes Montes de María desde sus capacidades productivas y económicas. Palobra, Palabra que obra, 15 (1).

Maza, F. (2016). Diseño de políticas públicas para la transformación de las prácticas agrícolas de la región del Canal del Dique y Zona Costera-Colombia-. Universidad de Cádiz.

Miyake, S.; Renouf, M.; Peterson, A.; McAlpine, C. \& Smith, C. (2012). Land-use and environmental pressures resulting from current and future bioenergy crop expansion: A review. Journal of Rural Studies. doi:10.1016/j.jrurstud.2012.09.002

Rathmann, R.; Szklo, A. \& Schaeffer, R. (2010). Land use competition for production of food and liquid biofuels: An analysis of the arguments in the current debate. Renewable Energy (35) 14-22. doi:10.1016/j.renene.2009.02.025

Ravindranath, N.; Lakshmi, C.; Manuvie, R. \& Manuvie, P. (2011). Biofuel production and implications for land use, food production and environment in India. Energy Policy. 39 (2011). 5737-5745. doi:10.1016/j.enpol.2010.07.044

SITMMA (2011). Sistema de información territorial de los Montes de María. Consultado el 0103-2012. 
WWF (2009). Palma de aceite. Documento de Posición WWF Colombia. Bogotá. Recuperado de http://www.wwf.org.co/?196011\%2FPalma-de-Aceite-Documento-posicin

Wakker, E. (2005). Gressy Palms. The social and ecological impacts of large-scale oil palm plantation development in Southeast Asia. Recuperado de http://www.foe.co.uk/resource/reports/ greasy_palms_impacts.pdf

Wicke, B.; Sikkema, R.; Dornburg, V. \& Faaij, A. (2010). Exploring land use changes and the role of palm oil production in Indonesia and Malaysia. Land Use Policy 28 (2011). 193-206. doi:10.1016/j.landusepol.2010.06.001 